\title{
Effect of GreenshellTM Mussel on the Osteoarthritis Biomarkers and Inflammation in Healthy Postmenopausal Women: A Study Protocol for Randomized Double - Blind Placebo - Controlled Trial
}

Maryam Abshirini ( $\square$ Maryam.Abshirini.1@uni.massey.ac.nz)

Massey University College of Health

Jane Coad

Massey University

Frances M. Wolber

Massey University

Pamela von Hurst

Massey University

Matthew R Miller

Cawthron Institute

Hong Sabrina Tian

Sanford Health

Marlena C Kruger

Massey University College of Health

Study protocol

Keywords: osteoarthritis, biomarker, inflammation, Greenshell mussel, Green-lipped mussel

Posted Date: January 12th, 2021

DOl: https://doi.org/10.21203/rs.3.rs-45327/v1

License: (c) (1) This work is licensed under a Creative Commons Attribution 4.0 International License.

Read Full License

Version of Record: A version of this preprint was published at Trials on July 28th, 2021. See the published version at https://doi.org/10.1186/s13063-021-05473-5. 


\section{Abstract}

Background: New Zealand Greenshell ${ }^{\text {TM }}$ mussels (GSM; Perna canaliculus) has recently been shown to decrease cartilage degradation in a rat model of induced metabolic osteoarthritis (MetOA). However, this effect has not been investigated in human subjects. This study aims to determine the effect of GSM powder on biomarkers of cartilage metabolism, bone resorption, and inflammation in New Zealand healthy overweight/obese postmenopausal women.

Method: Fifty overweight or obese (BMI $25-35 \mathrm{~kg} / \mathrm{m}^{2}$ ) postmenopausal women (aged 55-75 years) will be recruited. They will be randomly assigned to receive $3 \mathrm{~g} / \mathrm{d}$ whole meat GSM powder or placebo (sunflower seed protein) for 12 weeks. Data on socio-demographics, physical activity and dietary intake will be collected for each subject. Cartilage turnover biomarkers [(C-telopeptide of type II collagen (CTX-II), Cpropeptide of type II procollagen (CPII), Cartilage oligomeric matrix protein (COMP)], and bone resorption marker (CTX-I) will be measured in blood and urine samples. Inflammatory status (hs-CRP and cytokine panel) will be assessed and iron status will be measured. Body composition including fat mass (FM), lean mass (LM) and fat percentage will be measured using dual-energy X-ray absorptiometry (DXA). Joint pain and knee function will be assessed using a $100 \mathrm{~mm}$ Visual Analogue Scale (VAS) and the Knee injury and Osteoarthritis Outcome Score (KOOS) questionnaire, respectively.

Discussion: This trial will be the first to explore the effects of whole meat GSM powder on cartilage turnover, bone resorption and inflammation biomarkers in overweight/obese postmenopausal women. The results from this trial will provide evidence on the efficacy of GSM in prevention of OA.

Trial registration: The study was registered with the Australian New Zealand Clinical Trials Registry (ANZCTR) with the number ACTRN12620000413921p. Registration date: 27/03/2020. https://www.anzctr.org.au/Trial/Update/Step1_Update.aspx?id=379291

\section{Background}

Osteoarthritis $(\mathrm{OA})$ is the most common type of degenerative joint disease, contributing to progressive pain and functional loss. It is estimated to affect $33.6 \%$ of the elderly over 65 years of age $[1,2]$. OA affects an estimated $3.7 \%$ of the population worldwide and that equates to approximately 268 million people [3]. The public health burden of OA is increasing, in parallel with aging and obesity. OA is more prevalent in women compared to men, and the incidence is accelerated following menopause [4]. Estrogen depletion is an important risk factor for OA, possibly due to the presence of estrogen receptors (ERs) in joint tissues [5].

Menopausal transition is accompanied by weight gain which contributes to several health risks including musculoskeletal diseases [6]. An increased body mass index (BMI) is strongly related to an increased risk of knee and hip OA [7]. Excess body weight not only increases the OA risk at weight-bearing joints due to increased mechanical loading; it has been shown to increase the risk of OA in non-weight-bearing joints such as in the hands [8]. Increased adipose tissue secretes adipokines and pro-inflammatory cytokines 
contributing to low-grade systemic inflammation [9]. Tumor necrosis factor- alpha (TNF-a), interleukin-1 beta (IL-1 $\beta$ ), and interleukin- 6 (IL-6) play a crucial role in cartilage loss and bone resorption in the pathogenesis of $\mathrm{OA}$. These cytokines induce the production of cartilage degrading enzymes and suppress the synthesis of collagen type II and other cartilage matrix components [10].

So far, no therapeutic strategies have been proven yet and conventional treatments, particularly nonsteroidal anti-inflammatory drugs (NSAIDs), have been used to reduce inflammation and symptoms. Prolonged use of NSAIDs have been associated with adverse side effects such as gastric or peptic ulcers [11]. Hence, identification of safe interventions for disease prevention and treatment of early OA has become increasingly important. Green-lipped mussels (Perna canaliculus) or Greenshell ${ }^{\text {TM }}$ mussels (GSM) is a known commercial aqua cultured species native to New Zealand (NZ). GSM contains high amounts of long-chain omega-3 polyunsaturated fatty acids ( $n-3$ PUFA); eicosapentaenoic acid (20:5 n-3 EPA) and docosahexaenoic (22:6 n-3 DHA) and several other bioactive compounds [12]. With potent antiinflammatory properties, GSM oil extracts have shown to alleviate the arthritic symptoms [13].

The chondroprotective effect of GSM has recently been reported in an experimental model of OA. In this study, serum concentration of the cartilage degradation biomarker CTX-II was significantly reduced after GSM powder was added to the diet of rats actively developing metabolic OA [14]. Results from this preliminary study support the potential for an intervention study supplementing human subjects with GSM powder in order to slow the development of OA.

Obesity predisposes the individuals to subclinical inflammation and concurrently reduces iron absorption and systemic iron availability from cellular iron stores [15]. Obese people are at risk of developing iron deficiency, leading to escalation of the disease burden [16]. GSM contains a high concentration of both haem and non-haem iron as well as iron absorption enhancers including myofibrillar proteins, lowmolecular-weight aminoglycans and n-3 PUFA [17]. GSM enhanced the non-haem iron uptake in human intestinal epithelial cells (Caco-2) to a similar extent to that of beef [18]. For these reasons, we hypothesize that GSM could potentially improve the iron status biomarker.

To date, there has been no previous trial investigating the effect of GSM powder on OA biomarkers in human subjects. Therefore, the current clinical trial aims to investigate the effect of powder derived from New Zealand GSM on biomarkers of cartilage turnover, inflammation, body composition, and outcomes of joint pain and knee function in healthy overweight or obese postmenopausal women who are at great risk of developing $\mathrm{OA}$.

\section{Methods}

\section{Study design}

This is a randomized, double-blind, placebo-controlled trial. The flowchart of the study protocol is illustrated in Figure 1. This study will be conducted at Human Nutrition Research Unit at Massey University, Palmerston North, New Zealand. 


\section{Sample size}

The sample size calculation applies to CTX-II/ creatinine, CTX-II/ CPII ratio, and COMP as the primary outcome variables. For CTX-II/ creatinine as well as CTX-II/ CPII a sample size of 24 is required to detect a $20 \%$ difference from baseline with $80 \%$ power. Hence, 25 subjects will be recruited for each group (GSM and placebo). For COMP as well as CPII a sample size of 7 is required to detect a $20 \%$ difference from baseline.

\section{Study population}

Fifty apparently healthy postmenopausal women aged 55 to 75 years will be recruited by advertisement on campus and by using a recruitment agency, Trial Facts (https://trialfacts.com/). Written informed consent will be obtained from participants before commencing data collection.

\section{Inclusion and exclusion criteria}

For the present study, women who are at least five years postmenopausal (based on the natural cessation of menstruation), aged 55-75 years with no major illness, and body mass index (BMI) between 25 and 35 $\mathrm{kg} / \mathrm{m}^{2}$ will be included in the study.

Patients will be excluded if: 1) they have a formal diagnosis of OA or rheumatoid arthritis (RA); 2) have chronic liver or renal disease, diabetes mellitus and atherosclerosis; 3 ) have history of allergy to mussels or seafood; 4) history of joint injury or trauma; 5 ) smoke or have alcohol intake more than two units per day; 6) receiving supplements for joint health, multivitamins/mineral or omega-3 regularly and unwilling to stop these four weeks before beginning of the trial; 7) currently being on hormone replacement therapy or less than 6 months prior beginning the trial; 8) Continuously taking anti-inflammatory drugs or glucocorticoids or NSAIDs on a daily basis.

\section{Enrolment and washout period, randomization}

Eligible participants will be asked to stop consumption of oily fish (salmon, sardines, pilchards, tuna, etc.), and mussels four weeks before the beginning the trial until the end of trial (from weeks - 4, to week 12). After 4 weeks of washout, participants will be randomly allocated into two groups: whole meat GSM powder $(n=25)$ and placebo groups $(n=25)$ and will be followed-up for 12 weeks. Participants will be randomized based on a double-blind randomization schedule and stratified randomization will be used to match participants based on age and BMI distribution. Table 1 shows the schedule of enrollment, interventions, and assessments.

\section{Procedure}


After screening for eligibility, the informed consent form will be obtained and both groups will be invited to the Human Nutrition Research Unit at Massey University, Palmerston North three times: at baseline, week 6, and after 12 weeks. Subjects will be interviewed regarding their socio-demographic, dietary intake and physical activity at baseline. At each visit, fasting blood and urine samples will be collected. A venous blood sample $(20 \mathrm{ml})$ will be drawn after a 12-h overnight fast by a trained phlebotomist. Then blood samples will be centrifuged to obtain the serum and plasma EDTA and will be stored at $-80^{\circ} \mathrm{C}$ until further biochemical analyses. Body composition measurements including fat mass, lean mass and fat percentage will be measured and analyzed using the Hologic Horizon A, Dual energy X-ray Absorptiometry (DXA) at baseline and week 12 .

Serum cartilage biomarkers CTX-II, COMP and CPII will be assessed using commercially available sandwich enzyme immunoassay kits (BioVendor Research and Diagnostic Products, Karasek, Czech Republic). Bone marker CTX-I will be analyzed by electrochemiluminescence immunoassay using the Roche COBAS ${ }^{\circledR}$ e411 system (Roche Diagnostics, Indianapolis, IN, USA).

Urinary CTX-II will be measured using enzyme immunoassay (IDS immunodiagnostic System, Fountain Hills, Arizona, USA). Cytokine concentrations will be analyzed using BioLegend $\circledast$ LEGENDplex ${ }^{\mathrm{TM}}$ MultiAnalyte Flow Assay, following the manufacturer's instructions. To determine vitamin $D$ status at the baseline, calcidiol (25-hydroxyvitamin D [25(OH) D]) will be analyzed using isotope-dilution liquid chromatography-tandem mass spectrometry (ID-LC-MSMS) by Canterbury Health, Christchurch, New Zealand. Serum iron, soluble transferrin receptors, transferrin, and ferritin will be measured to assess the iron status by measured at MedLab Central Palmerston North, New Zealand to provide information on iron status. Urine creatinine will also be assessed by the colorimetric method at Human Nutrition Research Unit at Massey University, Palmerston North, New Zealand. Creatinine will be used to correct the level of urinary CTX-II according to the urinary concentration.

\section{Intervention}

Spray-dried GSM powder will be produced by Sanford Ltd (ENZAQ facility, Blenheim, New Zealand) using standard manufacturing processes. The proximate composition and microbiological load will be assessed by a commercial testing laboratory (Food Testing Laboratory of Cawthron Analytical Services; Nelson, New Zealand). The Association of Official Analytical Chemists (AOAC) methods for crude protein (AOAC 981.10), total fat (AOAC 948.15), moisture at $105^{\circ} \mathrm{C}$ (AOAC 950.46) and ash (AOAC 920.153) will be used and carbohydrate content will be determined by calculation ( $100 \%-\%$ crude protein - $\%$ total fat$\%$ moisture - \% ash). An aliquot of the total lipid extract from the GSM powder will be analyzed by gas chromatography mass spectrometry (GC-MS) according to AOAC 963.22. The placebo (sunflower seed protein powder) capsules will be purchased by Sanford Ltd from a commercial supplier. Both GSM and placebo with be encapsulated in gelatin capsules by a commercial facility (Alaron, Nelson NZ) and stored under nitrogen in the dark until use. Each capsule will contain $0.5 \mathrm{~g}$ of whole meat GSM powder or sunflower seed protein powder. The capsules will be completely similar in the shape, size and color 
(which will be dark to hide contents). Whole meat GSM powder and placebo capsules will be provided to both groups for 12 weeks. Participants will be required to consume 6 capsules ( 3 grams total) per day with meals.

\section{Compliance}

To assess subject's compliance during the 12 weeks, diaries will be provided at the randomization to record the compliance to dietary and medication/supplement restriction. At the week 6 and 12, subjects will return their unused capsules to researcher to assess the rate of the compliance. At weeks 6 and 12 consumption record diaries will be cross-checked with subjects. At the baseline and week 12, the plasma and erythrocyte membrane fatty acids will also be measured as indicator to assess the adherence to study protocol.

\section{Safety}

Subjects will be monitored closely during the study period and will be provided with daily diary to record all the medications taken and any occurrence of adverse events at weeks 6 and 12, the principal investigator will cross-check the recorded diaries with subjects. Routine laboratory measurements including liver and kidney function tests, blood glucose (non-fasting) and lipid profile (triglyceride, total cholesterol, HDL-cholesterol, LDL-cholesterol) will be performed at time of enrolment and after 12 weeks of treatment at MedLab Central Palmerston North, New Zealand by the certified phlebotomist. In case of any side effects or blood abnormalities, the person will be withdrawn and referred to their general practitioner for further examination.

\section{Study outcomes}

\section{Primary outcomes}

The changes in cartilage turnover markers; (CPII, COMP, CTX-II), bone resorption marker (CTX-I), measured at the end of the study in comparison with the week 6 and baseline values.

\section{Secondary outcomes}

The changes in inflammatory markers including hs-CRP and 13 cytokines namely IL-1 $\beta$, interferon alpha2 (IFN-a2), IFN- $\lambda$, TNF-a, monocyte chemoattractant protein-1 (MCP-1), IL-6, IL-8, IL-10, IL-12p70, IL-17A, IL-18, IL-23 and IL-33, as well as serum soluble transferrin receptor levels as an indicator of iron status measured at the end of the study in comparison with baseline values. Changes in body composition 
including fat mass (FM), lean mass (LM) and fat percentage, joint pain and knee function will also be measured.

\section{Assessment of dietary intake}

The New Zealand polyunsaturated fatty acids questionnaire will be applied to assess the habitual polyunsaturated fatty acids intake of participants after enrollment. To measure participants' dietary intake, a 3-day diet diary (3-DDD) over non-consecutive days (including one weekend day) will be collected three times during the study. The 3-DDD will be used to collect information on participants' food and beverage intake using household measurement tools to assist subjects in estimating the portion sizes of the food. Brand name of food products, recipes and method of food preparation will be described. Dietary intake will be analyzed with Food works 9 professional, Xyris software.

\section{Assessment of physical activity}

Physical activity will be assessed using the New Zealand Physical Activity Questionnaire-short form (NZPAQ-SF) [19]. The NZPAQ has previously been validated by Boon et al. Physical activity will be measured by METs-min/day which is computed by using the scoring protocol of IPAQ for continuous score [20]. MET values and formula for calculation of MET-minutes will be assessed and used as below:

- Walking MET-minutes/week at work $=3.3 \times$ walking minutes $\times$ walking days at work

- Moderate MET-minutes/week at work $=4.0 \times$ moderate-intensity activity minutes $\times$ moderate intensity days at work.

- Vigorous MET-minutes/week at work $=8.0 \times$ vigorous-intensity activity minutes $\times$ vigorous

- Total Work MET-minutes $/$ week = sum of Walking + Moderate + Vigorous MET-minutes $/$ week scores at work

\section{Assessment of joint pain and knee function}

Subjects will be provided an assessment of their joint pain level using the $100 \mathrm{~mm}$ Visual Analog Scale (VAS) at six-week intervals.

Knee Injury and Osteoarthritis Outcome Score (KOOS) questionnaire will be used to measure a subject's opinion about their knee and associated problems [21]. The KOOS consists of 42 questions, which cover five domains. These include 1) pain frequency and severity during functional activities; 2) severity of knee stiffness and swelling, grinding or clicking, catching, and limitation in range of motion; 3) difficulty in performing the daily living activities; 4) difficulty experienced during sport and recreational activities; and 5) knee-related quality of life (QOL). 
All items are scored on a 5-point Likert scale (0-4). The five dimensions are scored separately as the sum of all corresponding items. Scores are then converted to a 0-100 scale (percentage of total possible score obtained), where zero represents extreme knee problems and 100 represents no knee problems.

\section{Statistical analysis}

Statistical analysis will be performed using IBM statistics software version 25 (Armonk, NY, USA). The values of variables will be presented as mean \pm standard deviation. Normality tests will be assessed through Shapiro-Wilk tests carried out on each parameter before analysis. All outcome variables will be assessed based on per protocol analysis. To compare the mean differences between the two groups, independent t-test and Mann-Whitney will be used for normal and non-normal data respectively, considering the normality of data.

\section{Discussion}

To the best of our knowledge, this is the first randomized controlled trial that will determine the effect of whole meat GSM powder on cartilage metabolism, bone resorption, and inflammation biomarkers in overweight and obese postmenopausal women who at high risk of developing OA. The current study builds on a promising pre-clinical trial [14] which confirmed the chondroprotective effect of GSM powder in an experimental model of metabolic OA by reducing the CTX-II (decreasing type II collagen degradation) without any apparent adverse effects.

The current study aims to address several gaps in the current scientific evidence for GSM interventions. Firstly, until now there have been a limited number of studies which investigated the effectiveness of whole GSM extract interventions on clinical symptoms of OA in individuals with diseased state $[22,23]$. The current study will include biomarkers of cartilage turnover and inflammation to address the paucity of evidence on cartilage-protective effectiveness of GSM intervention in healthy individuals without OA. Secondly, this trial aims to target overweight and obese postmenopausal women, a population group more severely impacted by OA [24] and given the limited number of interventions in this population, this study will address this specific gap in the current evidence base.

To conclude, if the whole meat GSM intervention reduces the cartilage degradation and improves the cartilage synthesis, there is potential for the wide implementation of GSM powders as dietary supplements or functional foods, or even as a whole food to prevent or delay the onset of OA.

\section{Trial Status}

Recruitment will start in August 2020 and is planned to end in May 2021, with 50 participants enrolled. The current protocol version is 1.0, dated 17 July 2020 . 


\section{Declarations}

\section{Ethical considerations and consent to participate}

Massey University Human Ethics Committee approved this study: Southern A, Application 20/03. The informed consent form will be obtained from the participants. Whenever a subject is unable to continue the trial, she will be excluded from the study.

\section{Consent for publication}

Not applicable

\section{Availability of data and materials}

Not applicable

\section{Competing interests}

The authors declare that they have no competing interests

\section{Funding}

This study is funded by the National Science Challenge, High Value Nutrition (HVN) "Musseling-up 2.0: Greenshell ${ }^{T M}$ mussels for inflammation, metabolism and muscular skeletal function (HVN1904)", as a collaboration between Massey University, Cawthron Institute, Plant and Food Research Ltd, AgResearch and Sanford Ltd. HVN is underwritten by the Ministry of Business, Innovation and Employment (MBIE). The funder has no role or ultimate authority over any of the trial-related management, analysis, writing of the report, or the decision to submit the report for publication.

\section{Author's contribution}

MC.K: Conceptualization, methodology, supervision, M.A: Chief investigator, writing original draft preparation and protocol development. J.C, FM.W and P.H: Writing and reviewing and editing. MR.M and HS.T: Funding acquisition, review \& editing. All authors read and approved the final manuscript

\section{References}

1. Woolf AD, Pfleger B. Burden of major musculoskeletal conditions. Bull World Health Organ. 2003;81:646-56.

2. Lawrence RC, et al., Estimates of the prevalence of arthritis and other rheumatic conditions in the United States: Part Il. Arthritis \& Rheumatism, 2008. 58(1): p. 26-35.

3. Briggs AM, et al. Musculoskeletal health conditions represent a global threat to healthy aging: a report for the 2015 World Health Organization world report on ageing and health. Gerontologist. 2016;56(suppl_2):S243-55. 
4. Srikanth VK, et al. A meta-analysis of sex differences prevalence, incidence and severity of osteoarthritis. Osteoarthritis cartilage. 2005;13(9):769-81.

5. Richette P, Corvol M, Bardin T. Estrogens, cartilage, and osteoarthritis. Joint Bone Spine. 2003;70(4):257-62.

6. Kapoor E, Collazo-Clavell ML, Faubion SS. Weight gain in women at midlife: A concise review of the pathophysiology and strategies for management. in Mayo Clinic Proceedings. 2017. Elsevier.

7. Holliday $\mathrm{KL}$, et al. Lifetime body mass index, other anthropometric measures of obesity and risk of knee or hip osteoarthritis in the GOAL case-control study. Osteoarthritis Cartilage. 2011;19(1):37-43.

8. Oliveria SA, et al., Body weight, body mass index, and incident symptomatic osteoarthritis of the hand, hip, and knee. Epidemiology, 1999: p. 161-166.

9. Hauner $\mathrm{H}$, Secretory factors from human adipose tissue and their functional role. Proceedings of the Nutrition Society, 2005. 64(2): p. 163-169.

10. Wang T, He C. Pro-inflammatory cytokines: the link between obesity and osteoarthritis. Cytokine Growth Factor Rev. 2018;44:38-50.

11. Lanas A, et al. Risk of upper gastrointestinal bleeding associated with non-aspirin cardiovascular drugs, analgesics and nonsteroidal anti-inflammatory drugs. European Journal of Gastroenterology Hepatology. 2003;15(2):173-8.

12. Miller MR, et al. Regiospecific analyses of triacylglycerols of hoki (Macruronus novaezelandiae) and

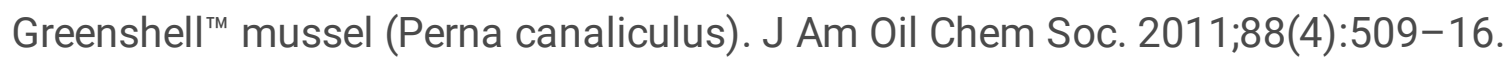

13. Coulson S, et al. Green-lipped mussel (Perna canaliculus) extract efficacy in knee osteoarthritis and improvement in gastrointestinal dysfunction: a pilot study. Inflammopharmacology. 2012;20(2):716.

14. Siriarchavatana P, et al. The Preventive Effects of Greenshell Mussel (Perna canaliculus) on EarlyStage Metabolic Osteoarthritis in Rats with Diet-Induced Obesity. Nutrients. 2019;11(7):1601.

15. Weiss G, Goodnough LT. Anemia of chronic disease. N Engl J Med. 2005;352(10):1011-23.

16. Zhao L, et al. Obesity and iron deficiency: a quantitative meta-analysis. Obesity reviews. 2015;16(12):1081-93.

17. Murphy K, Mann N, Sinclair A. Fatty acid and sterol composition of frozen and freeze-dried New Zealand Green Lipped Mussel (Perna canaliculus) from three sites in New Zealand. Asia Pac J Clin Nutr. 2003;12(1):50-60.

18. Stewart RJ, et al. New Zealand green-lipped mussels (Perna canaliculus) enhance non-haem iron absorption in vitro. British journal of nutrition. 2012;108(6):949-52.

19. McLean G, Tobias M, The New Zealand Physical Activity Questionnaires: Report on the validation and use of the NZPAQ-LF and NZPAQ-SF self-report physical activity survey instruments. 2004: SPARC.

20. Boon RM, et al. Validation of the New Zealand physical activity questionnaire (NZPAQ-LF) and the international physical activity questionnaire (IPAQ-LF) with accelerometry. Br J Sports Med. 
2010;44(10):741-6.

21. Roos EM, et al. Knee Injury and Osteoarthritis Outcome Score (KOOS)-development of a selfadministered outcome measure. Journal of Orthopaedic Sports Physical Therapy. 1998;28(2):88-96.

22. Coulson S, et al. Green-lipped mussel (Perna canaliculus) extract efficacy in knee osteoarthritis and improvement in gastrointestinal dysfunction: a pilot study. Inflammopharmacology. 2012;20(2):716.

23. Coulson S, et al. Green-lipped mussel extract (Perna canaliculus) and glucosamine sulphate in patients with knee osteoarthritis: therapeutic efficacy and effects on gastrointestinal microbiota profiles. Inflammopharmacology. 2013;21(1):79-90.

24. Hame SL, Alexander RA. Knee osteoarthritis in women. Curr Rev Musculoskelet Med. 2013;6(2):1827.

\section{Tables}

Table1. The schedule of enrolment, interventions, and assessments. 


\begin{tabular}{|c|c|c|c|c|c|}
\hline \multicolumn{6}{|c|}{ STUDY PERIOD } \\
\hline \multirow[b]{2}{*}{ TIMEPOINT } & \multirow{2}{*}{$\begin{array}{l}\text { Enrolment } \\
\text { Week - } 4\end{array}$} & \multirow{2}{*}{$\begin{array}{l}\text { Allocation } \\
\text { Week } 0\end{array}$} & \multirow{2}{*}{$\begin{array}{l}\begin{array}{l}\text { Post- } \\
\text { allocation }\end{array} \\
\qquad \text { Week } \\
1\end{array}$} & \multicolumn{2}{|c|}{$\begin{array}{l}\text { Study end } \\
\text { point }\end{array}$} \\
\hline & & & & $\begin{array}{l}\text { Week } \\
6 \\
\text { (follow } \\
\text { up) }\end{array}$ & $\begin{array}{l}\text { Week } \\
12\end{array}$ \\
\hline \multicolumn{6}{|l|}{ ENROLMENT: } \\
\hline Eligibility screen & $\mathrm{x}$ & & & & \\
\hline Informed consent & $x$ & & & & \\
\hline Washout & $x$ & & & & \\
\hline Allocation & & $x$ & & & \\
\hline \multicolumn{6}{|l|}{ INTERVENTIONS: } \\
\hline Green shell mussel & & & $x$ & $x$ & $x$ \\
\hline placebo & & & $x$ & $x$ & $x$ \\
\hline \multicolumn{6}{|l|}{ ASSESSMENTS: } \\
\hline $\begin{array}{l}\text { Demographic assessment, physical } \\
\text { activity }\end{array}$ & & $x$ & & & \\
\hline Cartilage marker, bone marker & & $x$ & & $x$ & $x$ \\
\hline $\begin{array}{l}\text { Inflammatory marker, body } \\
\text { composition, iron stats marker, join } \\
\text { pain and knee pain }\end{array}$ & & $x$ & & & $x$ \\
\hline
\end{tabular}

Figures 


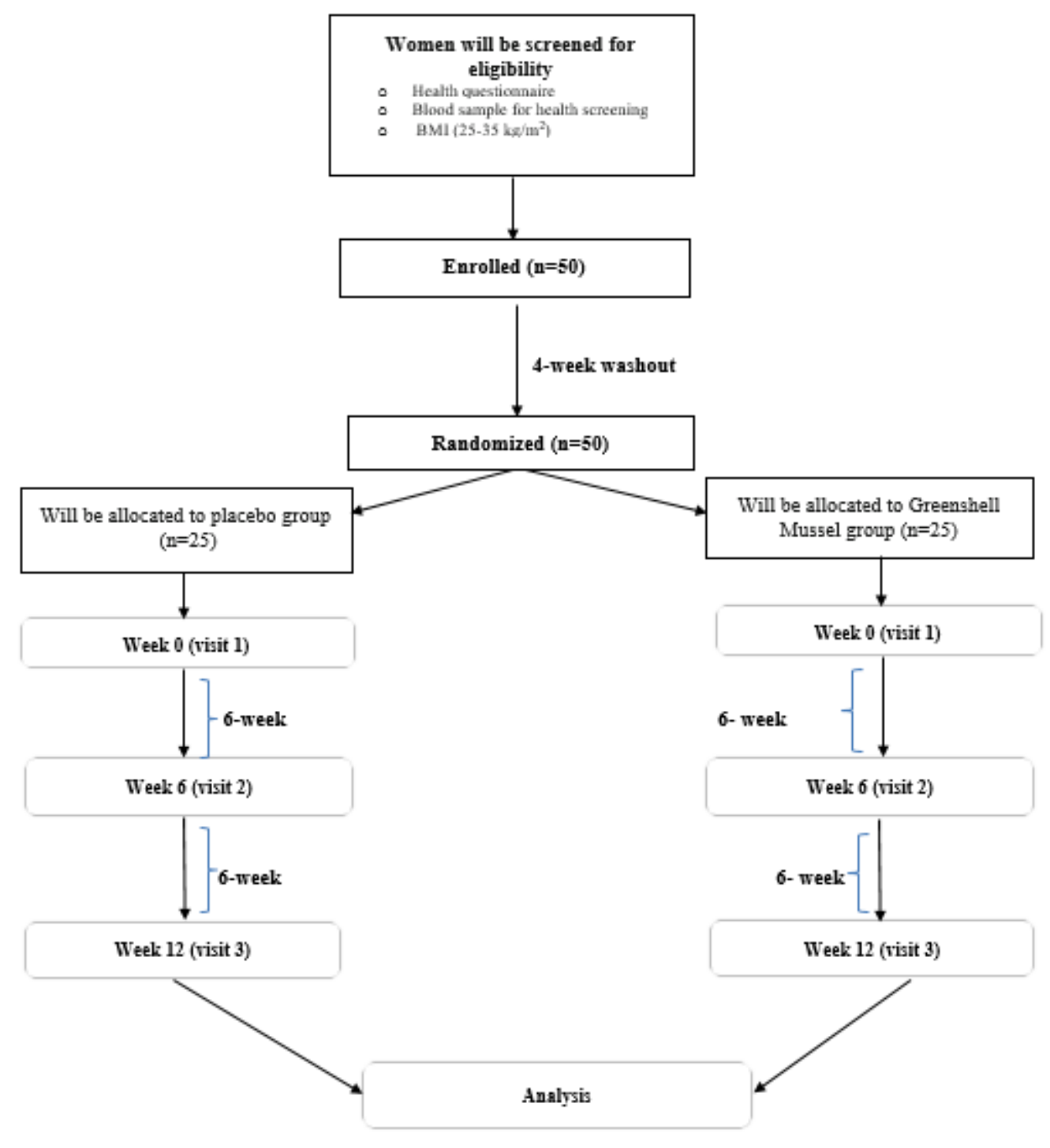

Figure 1

Flowchart describing the study

\section{Supplementary Files}

This is a list of supplementary files associated with this preprint. Click to download.

- SPIRITFillablechecklist15Aug2013.doc 